\title{
Magnetic and Structural Studies into the Effect of Solvent Exchange Process in Metal-Organic Framework MOF-76(Gd)
}

\author{
M. Almášs ${ }^{a, *}$, V. ZeleñáKK ${ }^{a}$ AND A. ZeleñÁKOVÁ ${ }^{b}$ \\ ${ }^{a}$ Institute of Inorganic Chemistry, Faculty of Science, P.J. Šafárik University, Moyzesova 11, 04154 Košice, Slovakia \\ ${ }^{b}$ Institute of Physics, Faculty of Science, P.J. Šafárik University, Park Angelinum 9, 04154 Košice, Slovakia

\begin{abstract}
Effect of solvent exchange/absence on magnetic and structural properties of microporous metal-organic frame-
\end{abstract} \\ work MOF-76(Gd) has been performed. Three compounds have been prepared and characterized: as synthesized \\ MOF-76(Gd)-DMF, which containing DMF ( $N, N^{\prime}$-dimethylformamide) and $\mathrm{H}_{2} \mathrm{O}$ molecules in the cavity system, \\ activated complex without solvents, MOF-76 $(\mathrm{Gd})$ and water exchanged sample MOF-76(Gd)- $\mathrm{H}_{2} \mathrm{O}$. The structural \\ study showed that compounds undergo structural changes after activation/exchange processes. Changes were \\ mainly observed in the magnetic properties and distances between Gd...Gd ions: $4.718 \AA$ for MOF-76(Gd)-DMF, \\ $4.326 \AA$ for MOF-76(Gd)- $\mathrm{H}_{2} \mathrm{O}$ and $4.296 \AA$ for MOF-76(Gd). The magnetic properties of the samples were stud- \\ ied by magnetic susceptibility $\chi_{M}(T)$ and magnetization $M(H)$. The low positive value of the Weiss constant, \\ $\Theta=1.54 \mathrm{~K}$ in compound MOF-76(Gd)-DMF shows on a very weak ferromagnetic interaction. On the contrary, the \\ negative values of $\Theta$ in complexes MOF-76(Gd) $(-5.58 \mathrm{~K})$ and MOF-76 $(\mathrm{Gd})-\mathrm{H}_{2} \mathrm{O}(-6.23 \mathrm{~K})$ confirm the existence \\ of antiferromagnetic exchange interaction between $\mathrm{Gd}(\mathrm{III})$ ions.
}

DOI: 10.12693/APhysPolA.131.991

PACS/topics: 75.30.Sg, 75.75.Fk, 65.40.gd

\section{Introduction}

The assembly of organic molecules and metal ions may yield novel types of $3 \mathrm{D}$ networks that contain channels with various sizes and shapes and which are denoted as metal-organic frameworks (MOFs). MOFs based on lanthanide metal ions or clusters represent a group of ultra-highly-porous materials that possess interesting coordination, catalytic, adsorption and magnetic properties $[1,2]$.

One of the intensively studied porous isostructural lanthanide-based frameworks is MOF-76, which consists of lanthanide ions and benzene-1,3,5-tricarboxylate linker (BTC). These 3D transformable frameworks exhibit permanent porosity and extremely high thermal stability. In our previous work, we have investigated stability and applications of MOF-76 containing $\mathrm{Ce}(\mathrm{III})$, Ho(III), Tm(III) and Lu(III) cations [3, 4]. Here we continue in our research with gadolinium(III) form namely, $\left\{\left[\mathrm{Gd}(\mathrm{BTC})\left(\mathrm{H}_{2} \mathrm{O}\right)\right] \cdot \mathrm{DMF}\right\}_{n}$. These frameworks contain 1D shaped channels filled with DMF molecules, which could be removed by the thermal treatment.

It is well known that magnetic exchange interactions are traditionally established through direct and super-exchange mechanisms between metal centers or metal centers and various ligands. We have investigated magnetic properties of three samples: as synthesized (containing DMF and water in the cavity system), activated (without solvent) and water exchanged $\left\{\left[\mathrm{Gd}(\mathrm{BTC})\left(\mathrm{H}_{2} \mathrm{O}\right)\right] \cdot 2 \mathrm{H}_{2} \mathrm{O}\right\}_{n}$. We have studied the effect

*corresponding author; e-mail: miroslav.almasi@upjs.sk of solvent absence/exchange on magnetic properties using a SQUID based magnetometer in external $d c$ field up to $5 \mathrm{~T}$ in the temperature range of $2-300 \mathrm{~K}$.

\section{Experimental}

Compound MOF-76(Gd)-DMF with composition $\left\{\left[\mathrm{Gd}(\mathrm{BTC})\left(\mathrm{H}_{2} \mathrm{O}\right)\right] \cdot \mathrm{DMF}_{\mathrm{n}}\right.$ was synthesized by solvothermal route at $80^{\circ} \mathrm{C}$ in the mixture of solvents $\mathrm{H}_{2} \mathrm{O} / \mathrm{EtOH} / \mathrm{DMF}[3,4]$. Activated sample, $\{[\mathrm{Gd}(\mathrm{BTC})]\}_{\mathrm{n}}$, without DMF and $\mathrm{H}_{2} \mathrm{O}$ solvents in the cavity system was prepared by heating of as synthesized complex at $400^{\circ} \mathrm{C}$ in an oven and the sample was denoted as MOF-76(Gd). Water exchanged sample MOF$76(\mathrm{Gd})-\mathrm{H}_{2} \mathrm{O}$ with formulae $\left\{\left[\mathrm{Gd}(\mathrm{BTC})\left(\mathrm{H}_{2} \mathrm{O}\right)\right] \cdot 2 \mathrm{H}_{2} \mathrm{O}\right\}_{\mathrm{n}}$ was prepared from activated form by its dispersion in distilled water.

Single crystal X-ray measurements were performed on a Xcalibur four-circle CCD diffractometer equipped with a graphite monochromator and using Mo $K_{\alpha}$ radiation $(\lambda=0.71073 \AA)$.

High-energy powder X-ray diffraction (HE-PXRD) measurements were carried out at P02.1 undulator beamline of PETRA III electron storage ring at DESY (Hamburg, Germany). The patterns were collected during in situ heating with heating rate $10^{\circ} \mathrm{C} \mathrm{min}^{-1}$ and radiation wavelength $\lambda=0.20715 \AA$.

Thermal behavior was studied by the combination of thermogravimetry (TG) and differential thermal analysis (DTA) using STA Netzsch 409 PC apparatus in atmosphere of argon with heating rate $9^{\circ} \mathrm{C} \mathrm{min}^{-1}$.

Temperature dependence of molar susceptibility was obtained by SQUID based magnetometer MPMS 5XL (Quantum Design) in the temperature range 1.8-300 K on three samples MOF-76(Gd)-DMF, MOF-76(Gd) and $\mathrm{MOF}-76(\mathrm{Gd})-\mathrm{H}_{2} \mathrm{O}$ in both regimes zero field cooled 
and field cooled (ZFC/FC) in magnetic field $100 \mathrm{mT}$. The powder specimens (20.6 mg for MOF-76(Gd)-DMF, $20.2 \mathrm{mg}$ for MOF-76(Gd), and $17.8 \mathrm{mg}$ for MOF-76(Gd)$\left.\mathrm{H}_{2} \mathrm{O}\right)$ were fixed in a gelatine capsule and the capsule was held by a straw. The signal contribution of empty gelcap and the straw was subtracted from the total signal. Also, the obtained data were corrected for the diamagnetic contribution using the Pascal constants [5].

\section{Results and discussion}

The crystal structures of prepared samples are depicted in Fig. 1. In general, compounds crystallize in tetragonal space group $P 4_{1} 22$ and their structures are constructed from $\mathrm{Gd}(\mathrm{III})$ ions, which are bridged by BTC organic linkers to form a neutral three-dimensional rodpacking structure. Each gadolinium atom is coordinated by five oxygen atoms from the carboxylate groups of BTC and further capped by one coordinated water molecule. As can be seen from Fig. 1a, one BTC molecule simultaneously bridges six different gadolinium ions, which are packed into the helical strands. The final crystal structures of studied compounds: as synthesized sample MOF-76(Gd)-DMF, activated MOF-76(Gd) and water exchanged sample $\mathrm{MOF}-76(\mathrm{Gd})-\mathrm{H}_{2} \mathrm{O}$ are shown in Fig. 1b-d. Frameworks contain approximately $6.8 \times$ $6.8 \AA^{2}$ one-dimensional sinusoidally shaped channels propagating along $c$ axis, which are non-occupied or filled with crystallization solvent molecules (DMF and $\left.\mathrm{H}_{2} \mathrm{O}\right)$.
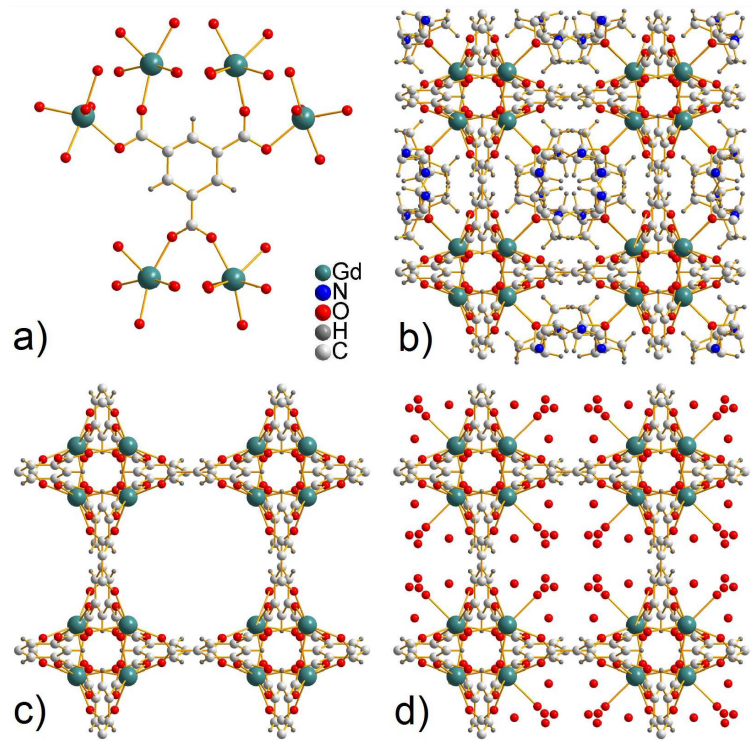

Fig. 1. (a) Coordination mode of BTC linker in MOF$76(\mathrm{Gd})$ samples. View of the final crystal structure of (b) as synthesized MOF-76(Gd)-DMF, (c) activated MOF-76(Gd), and (d) water exchanged compound $\mathrm{MOF}-76(\mathrm{Gd})-\mathrm{H}_{2} \mathrm{O}$.

The interesting feature of presented complexes is the presence of helical chains formed from $\mathrm{GdO}_{6}$ polyhedrons propagating along $c$ axis, which lie on the screw axis $4_{1}$. The shortest distances between two Gd(III) ions in the helical strands are: $4.718 \AA$ for MOF-76(Gd)DMF, $4.296 \AA$ for MOF-76(Gd) and $4.326 \AA$ for MOF$76(\mathrm{Gd})-\mathrm{H}_{2} \mathrm{O}$.

To estimate the thermal stability and solvent removal from the framework of as synthesized sample MOF76(Gd)-DMF, TG-DTA and HE-PXRD measurements were performed. It was shown that the desolvation process takes place in the temperature range $80-400^{\circ} \mathrm{C}$ and activated form is then thermally stable after heating up to $600{ }^{\circ} \mathrm{C}$.

HE-PXRD experiments performed during in situ heating in the temperature range $30-600^{\circ} \mathrm{C}$ using synchrotron radiation were used to investigate the framework stability. As can be seen from Fig. 2, under heating of MOF-76(Gd)-DMF, two phase structure transitions were observed corresponding to phenomenon called "singlecrystal-to-single-crystal transformation" (SCSC) [3, 4]. After heating, framework undergoes structural changes upon removal of solvent molecules from tetragonal (20$\left.140^{\circ} \mathrm{C}\right)$ to monoclinic $\left(150-320^{\circ} \mathrm{C}\right)$ phase and at higher temperature back to tetragonal crystallographic system $\left(330-600^{\circ} \mathrm{C}\right)$.

Crystallinity and structural integrity of the frameworks after solvent removal/exchange were also monitored using HE-PXRD (see inset of Fig. 2). Patterns are almost identical, evidencing the phase purity of the samples. Small differences in the peak positions and intensities could be explained by the fact that frameworks are transformable and presence or absence of solvents in the cavity system have a significant influence on the final shortest Gd...Gd distances, described above.

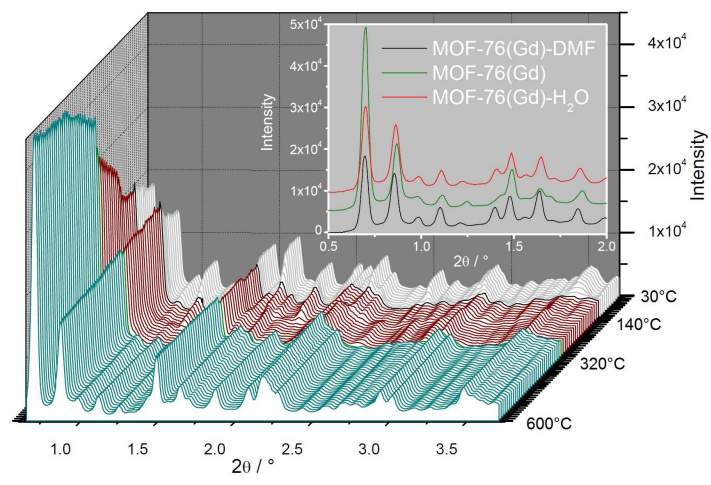

Fig. 2. The HE-PXRD patterns of as synthesized sample measured during in situ heating, in the temperature range $30-600^{\circ} \mathrm{C}$. Inset shows the HE-PXRD patterns of studied compounds.

To compare the magnetic properties of three different MOF systems, the molar magnetic susceptibility of complexes was measured in the range of $1.8-300 \mathrm{~K}$ in the magnetic field of $100 \mathrm{mT}$. Magnetic susceptibility data, recorded in both ZFC and FC regimes are shown in Fig. 3. The temperature dependence of inverse magnetic susceptibility (inset of Fig. 3) perfectly follows the Curie-Weiss law and a linear fit for temperature $20-300 \mathrm{~K}$ gives values of the Weiss constant, $\Theta=1.54 \mathrm{~K}$ for MOF-76(Gd)- 
DMF, $\Theta=-6.23 \mathrm{~K}$ for MOF-76(Gd), and $\Theta=-5.58 \mathrm{~K}$ for MOF-76(Gd)- $\mathrm{H}_{2} \mathrm{O}$. No difference between the magnetic response of the field cooled (FC) and zero-fieldcooled (ZFC) regimes in studied samples was observed, indicating no transition to the long-range ordered state in studied magnetic systems.

The low positive value of $\Theta$ in compound MOF$76(\mathrm{Gd})$-DMF shows on a very weak ferromagnetic (FM) interaction. On the contrary, the negative values of $\Theta$ in complexes MOF-76(Gd) and $\mathrm{MOF}-76(\mathrm{Gd})-\mathrm{H}_{2} \mathrm{O}$ confirm the presence of antiferromagnetic exchange interaction between Gd(III) ions. The ferromagnetic behavior was also observed by [6] on gadolinium-based metal-organic framework $\left\{\left[\mathrm{Gd}(\mathrm{EDA})_{1.5}\left(\mathrm{H}_{2} \mathrm{O}\right)_{2}\right] \cdot \mathrm{DMF}\right\}_{\mathrm{n}}$ $(\mathrm{EDA}=$ (ethylenedithio)acetate). The density functional theory (DFT) calculations confirm that the ferromagnetic properties in [7] originated from the $4 f$ electrons of $\mathrm{Gd}(\mathrm{III})$ propagating by a super-exchange pathway on two $-/+/-$ spin nets of the carboxylate groups.

The parameters of the exchange interactions calculated for presented three samples gives $J / k_{\mathrm{B}}=0.3 \mathrm{~K}$ for MOF$76(\mathrm{Gd})-\mathrm{DMF}, J / k_{\mathrm{B}}=-1.8 \mathrm{~K}$ for $\mathrm{MOF}-76(\mathrm{Gd})$, and $J / k_{\mathrm{B}}=-1.06 \mathrm{~K}$ for MOF-76(Gd)- $\mathrm{H}_{2} \mathrm{O}$.

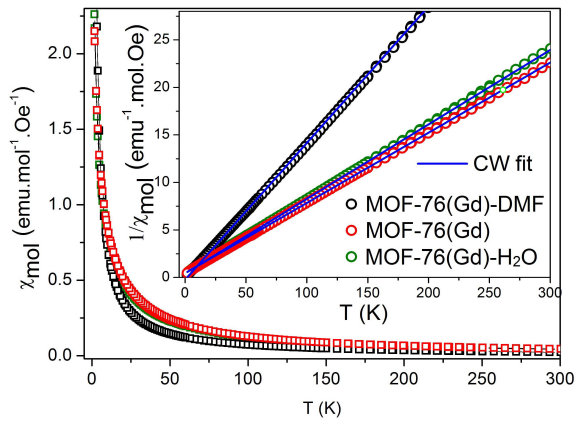

Fig. 3. Molar magnetic susceptibility depending on temperature. Inset shows the reciprocal value of molar magnetic susceptibility with a linear fit according to the Curie-Weiss law.

Figure 4 shows the comparison of the temperature dependence of the effective magnetic moment $\mu_{e f f}$ per the Bohr magnetron $\mu_{\mathrm{B}}$ for prepared samples. Effective magnetic moment per formula unit at room temperature $7.47 \mu_{\mathrm{B}}$ is close to theoretical value $7.94 \mu_{\mathrm{B}}$ for MOF-76(Gd)-DMF. Otherwise, the higher values $10.3 \mu_{\mathrm{B}}$ and $9.9 \mu_{\mathrm{B}}$ were estimated from experimental data for MOF-76(Gd) and MOF-76 $(\mathrm{Gd})-\mathrm{H}_{2} \mathrm{O}$, respectively. A decrease of $\mu_{\text {eff }}$ below $50 \mathrm{~K}$ (Fig. 4) in MOF-76(Gd) and $\mathrm{MOF}-76(\mathrm{Gd})-\mathrm{H}_{2} \mathrm{O}$ is observed, suggesting the presence of stronger AF exchange interaction.

\section{Conclusions}

In the present study, three compounds with general composition MOF-76(Gd)-X (X $=$ DMF, $\mathrm{H}_{2} \mathrm{O}$, no solvent) were prepared, characterized and their structural comparing to magnetic properties were investigated. The magnetic studies of prepared samples showed on a very weak ferromagnetic interaction for MOF-76(Gd)-DMF

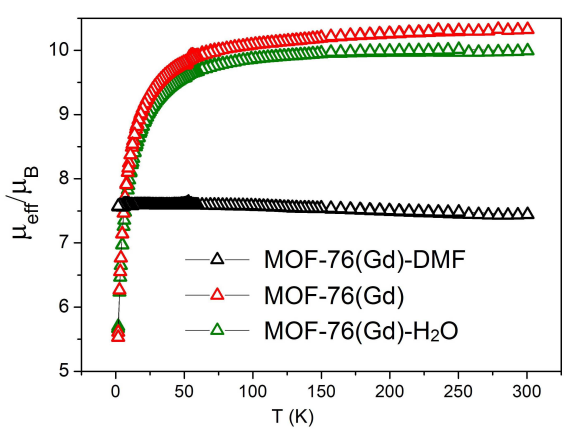

Fig. 4. Temperature dependence of the effective magnetic moment: comparison of experimental data measured on three samples, MOF-76(Gd)-DMF - containing DMF and water in the cavity system, MOF-76(Gd) - without solvent in the cavity and MOF-76(Gd)- $\mathrm{H}_{2} \mathrm{O}$ - water exchanged MOF-76(Gd).

$\left(\Theta=1.54 \mathrm{~K}, J / k_{\mathrm{B}}=0.3 \mathrm{~K}\right)$ and antiferromagnetic exchange interactions in the MOF-76(Gd) $(\Theta=-6.23 \mathrm{~K}$, $\left.J / k_{\mathrm{B}}=-1.8 \mathrm{~K}\right)$ and MOF-76(Gd)- $\mathrm{H}_{2} \mathrm{O}(\Theta=-5.58 \mathrm{~K}$, $\left.J / k_{\mathrm{B}}=-1.06 \mathrm{~K}\right)$. Moreover, effective magnetic moment per formula unit $7.47 \mu_{\mathrm{B}}$ is close to theoretical value $7.94 \mu_{\mathrm{B}}$ for MOF-76(Gd)-DMF. On the other hand, the values $10.3 \mu_{\mathrm{B}}$ and $9.9 \mu_{\mathrm{B}}$ estimated for MOF-76(Gd) and MOF-76(Gd)- $\mathrm{H}_{2} \mathrm{O}$ are significantly higher and also confirmed the presence of antiferromagnetic exchange interaction. Observed results could be attributed to different distances between $\mathrm{Gd}$...Gd ions in the framework of the samples: $4.718 \AA$ for MOF-76(Gd)-DMF, $4.296 \AA$ for MOF-76(Gd) and $4.326 \AA$ for MOF-76(Gd)- $\mathrm{H}_{2} \mathrm{O}$. The detailed analysis of paths of the magnetic exchange interactions will be performed in the near future.

\section{Acknowledgments}

This work was supported by the Slovak Research and Development Agency under the contracts APVV-0073-14 and APVV-520-15, by the VEGA project no. 1/0745/17 and project from P.J. Šafárik University no. VVGS-2016249. Part of this research was carried out at the light source PETRA III (beamline P02.1) at DESY, a member of the Helmholtz Association (HGF).

\section{References}

[1] H. Furukawa, K.E. Cordova, M. O'Keeffe, O.M. Yaghi, Science 341, 1230444 (2013).

[2] M. Almáši, A. Zeleňáková, I. Císařová, J. Bednarčík, V. Zeleňák, Acta Phys. Pol. A 126, 308 (2014).

[3] M. Almáši, V. Zeleňák, M. Opanasenko, I. Císařová, Catal. Today 243, 3098 (2015).

[4] M. Almáši, V. Zeleňák, J. Kuchár, S. Bourrelly, P.L. Llewellyn, Col. Surf. A Physicochem. Eng. Asp. 496, 114 (2016).

[5] G. Bain, J.F. Berry, J. Chem. Educat. 85, 532 (2008).

[6] G.I. Zhuang, L. Tan, W. Chen, J. Bai, X. Zhong, J. Wang, Cryst. Eng. Commun. 30, 6963 (2014).

[7] P. Poddar, S. Srinath, J. Gass, B.L.V. Prasad, H. Srikanth, J. Phys. Chem. C 111, 14060 (2007). 